\title{
Automated Functional and Behavioral Health Assessment of Older Adults with Dementia
}

\author{
Mohammad Arif Ul Alam ${ }^{1}$, Nirmalya Roy ${ }^{1}$, Sarah Holmes ${ }^{2}$, Aryya Gangopadhyay ${ }^{1}$, Elizabeth Galik ${ }^{3}$ \\ ${ }^{1}$ Department of Information Systems, University of Maryland, Baltimore County, MD, USA \\ ${ }^{2}$ Department of Gerontology, University of Maryland, Baltimore and Baltimore County, MD, USA \\ ${ }^{3}$ School of Nursing, University of Maryland, Baltimore, MD, USA \\ alam4@umbc.edu,nroy@umbc.edu, sarah65@umbc.edu,gangopad@umbc.edu, galik@son.umaryland.edu
}

\begin{abstract}
Dementia is a clinical syndrome of cognitive deficits that involves both memory and functional impairments. While disruptions in cognition is a striking feature of dementia, it is also closely coupled with changes in functional and behavioral health of older adults. In this paper, we investigate the challenges of improving the automatic assessment of dementia, by better exploiting the emerging physiological sensors in conjunction with ambient sensors in a real field environment with IRB approval. We hypothesize that the cognitive health of older individuals can be estimated by tracking their daily activities and mental arousal states. We employ signal processing on wearable sensor data streams (e.g., Electrodermal Activity (EDA), Photoplethysmogram (PPG), accelerometer (ACC)) and machine learning algorithms to assess cognitive impairments and its correlation with functional health decline. To validate our approach, we quantify the score of machine learning, survey and observation based Activities of Daily Living (ADLs) and signal processing based mental arousal state, respectively for functional and behavioral health measures among 17 older adults living in a continuing care retirement community in Baltimore. We compare clinically observed scores with technology guided automated scores using both machine learning and statistical techniques.
\end{abstract}

Keywords-activity recognition, cognitive analysis, pervasive computing, signal processing

\section{INTRODUCTION}

Dementia in the older adult population is one of the biggest global public health and social care challenges. In United states, approximately 5.4 million people of all ages have dementia, whereas 5.2 million are 65 and older. This number will continue to rise as the percentage of the older population increases. Nearly 13 percent of those aged $65+$ and almost 50 percent of the $85+$ population has dementia [1]. However, dementia is one of the most expensive diseases in developed countries, and its prevalence is expected to double within the next 20 years [2]. Although clinical and diagnostic tests have become more precise in identifying dementia, studies have shown that there is a high degree of underrecognition. Those studies have shown that in more than 50 percent of the cases, it is the family members who serve as the source of primary recognition and not the general practice health care providers [7]. Consequently, early detection and automatic disease prediction with high accuracy is needed, as early recognition and treatment may positively impact the course of the disease, the well-being and safety of the individuals and may attenuate dementia's economic impact on society. The use of technology in developing systems that promote older adults' independence and aging-in-place has been well received by many caregivers, clinicians, older adults, and family members [3], [14]. The maturing ubiquitous computing technologies enable extraordinary improvement of early detection of dementia in aging population [3]. Though cognitive impairment has significant correlations with everyday task performance [3], [14], we argue that combining mental arousal state with task will significantly improve the dementia prediction. Continuous mental arousal and activity quality monitoring help people better understand their mental and functional behavioral patterns and provide health care providers with more reliable data for interventions. Prior studies on mental arousal and activity monitoring were focused on observational monitoring systems in a laboratory environment where participants generally rested in a sedentary position [22]. In this study, we aim to monitor mental arousal and functional activities in participants' natural living environments, free from any artificial laboratory setup, engagement in a new environment or outer interventions.

With recent advances in wearable biosensing technologies, it is now feasible to develop systems that automatically monitor not only outwardly observable behaviors, but also inward physiological states of older adults, which may also serve as a physiological marker for their impairment status. Such technologies may not only help characterize qualitative aspects of older adult's functional ability but may potentially also assist with the early identification and quantification of dementia symptoms. A number of physiological markers are extensively used for continuous arousal measurement including galvanic skin response (GSR), heart beat patterns, blood pressure (BP), and respiration activity etc [6], [22]. There are also a multitude of physical changes such as increased heart rate, quickened reflexes, face pattern changes (eyebrow, nose, cheeks etc.), exhilaration of breathing rate [5]. Ubiquitous availability of miniaturized wireless devices capable of monitoring these physiological markers and physical changes helps people to closely track changes in their vital signs in order to detect and better manage any cognitive and functional health decline. However, measuring physiological signals during everyday activity is more difficult than in a rigorous laboratory environment. The physiological responses caused by mental arousal can be masked by variations due to physical activity aiding in the continuous monitoring of mental stress level [8].

In this paper, we rely on leveraging physiological sensors and smart home technologies to identify older adults who may have been clinically assessed for cognitive impairment by well established survey tools. In particular, we monitor the physiological signals (such as electrodermal activity) and 
activities of daily living (ADLs) performances of 17 older adults (6 with dementia, 3 with mild cognitive impairment, and 8 without cognitive impairment) during a scripted activity sessions. In addition, we employ a mixture of supervised and unsupervised machine learning approaches to automatically differentiate older adults with cognitive impairment (severe or mild) versus healthy (normal). Unlike the previous researches [3], [14], we focus on a field study rather than an artificial lab study, setting up smart home system in each participant's living environment to collect naturalistic physiological and activity data. We also build a low-cost plug-n-play smart home PogoPlug [35] system to deploy in a continuing care retirement community across multiple units.

\section{Research Challenges:}

- Physiological markers can be easily affected by physical activities and movements. For example, one physiological marker of arousal detection is increased heart rate which can also be noticed for walking or running state than sitting state. Additionally, heart rate can be higher during eating activity than resting although both of the complex activities are composed of postural event (sitting). Therefore using heart rate alone as an indicator to detect mental arousal may lead to misclassification. However, the subjects may demonstrate different types of arousal responses against the same complex activities based on their current arousal level, personality, age and mental disorders. Thus, designing signal processing and machine learning algorithms capable of tackling above challenges in the real environment are of utmost importance for validating the technology-based assessment being comparable or outperforming the observation-based clinical assessment.

- One of the major functional performance measures is activity duration, though that can be highly affected by the cognitive and physical ability of individuals. For example, a physically fit older adult with dementia may take less time to make sandwiches than older adults without dementia who has impaired physical ability obfuscating the automated assessment methodologies.

- Instrumenting and deploying sensor-based smart home systems at each participant's natural living environment warrants for assembling a flexible set of hardware and software interfaces to ease the system configuration, setup, and network discovery processes. This implementation requires flexible networking protocol setup that works in any secured network and remote monitoring facilities. However, the sensor system placed in the residences of volunteers needs to meet several specific physiological signals and activity monitoring needs. While their basic function is to collect and communicate sensor telemetry related to occupant's activity, we must confirm that the devices are reliable with potential for re-deployment as well as appear unintimidating to the participants. Motivated by these shortcomings, we propose to develop an autonomous functional and behavioral health assessment framework suited for the older adults in their everyday living environment.

Our research attempts to answer the following research questions:

- What advances in signal processing and machine learning techniques are required for automated longitudinal health assessment of older adults with dementia combining both physiological signals and activity performance measures?
- Is there any mutual statistical correlation between functional and cognitive health of older adults? If so what are the critical roles of survey-based (such as Barthel [34] and IADL scale [19], Zung anxiety [18]) observation-based (observation of the scripted activities and the SLUMS [23] to directly measures their cognitive performance) and technology guided assessment methodologies and their inter-relationships?

Key Contributions: The main contributions of our work are summarized below.

- We demonstrated how a signal processing approach augmented with machine learning techniques can be employed to discriminate older adults with dementia and mild cognitive impairment from their cognitively healthy counterparts using predefined measures associated with subjects' activity performances and physiological signals (such as EDA and PPG based mental arousal features).

- We depicted the group correlations between cognitively healthy and impaired older adults and articulated the effectiveness of technology, survey and observation-based cognitive and functional health assessment methodologies and their mutual co-relations, benefits and disadvantages in a clinical experimental setting.

- We collected 17 older adults data in a continuing care retirement community center in Baltimore city over a 5 months study time in contrast to a majority of the existing work where data have been obtained by carrying out the activities in a laboratory setting or smart home on-campus that might not have necessarily reflected the real cognitive and functional health status and performance of the subjects. We also design an integrated hardware and software interfaces of a lowcost plug-n-play smart home system [35] with commercially available off-the-shelf sensor devices [20] and a wearable smart wristband (Empatica E4 with EDA, PPG and ACC sensors on board) [41]. All of the relevant devices, the integrated smart home system and study methodologies were approved by a University Institutional Review panel and appropriate consent and assent procedures were followed prior to enrolling participants in the study.

\section{BACKGROUND ON PHYSIOLOGICAL SignAls}

The autonomic nervous system (ANS) restrains the body's major physiological activities including the heart rate, gland secretion, blood pressure, and respiration. The ANS is divided into sympathetic (SNS) and parasympathetic (PNS) branches. The SNS actuates the body's resources for action under arousal conditions. On the contrary, PNS attenuates the body and helps preserve the body regaining the steady state [8].

Arousal Relation with EDA and HR: Electrodermal activity (EDA) is the property of the human body that causes continuous variation in the electrical characteristics of the skin. The traditional theory of EDA holds that skin resistance varies with the state of sweat glands in the skin. Sweating is controlled by the sympathetic nervous system (SNS) [9] and skin conductance is an indication of psychological or physiological arousal. If SNS is highly aroused, then sweat gland activity also increases, which in turn increases skin conductance. In this way, skin conductance can be a measure of emotional and sympathetic responses. More recent research and additional 
phenomena (resistance, potential, impedance, and admittance) suggest that more thorough investigation is needed, and thus research continues into finding the source and significance of EDA [10]. On the other hand, SNS activation increases heart rate whereas PNS activation decreases it [11]. During the last few decades, researchers have also used Heart rate variability (HRV) to measure mental stress [11]. This important parameter provides a window through which the heart's ability to respond to normal regulatory impulses can be observed. HRV measures are calculated from the tachogram, also called RR interval time series. These data are derived from the ECG signal by defining the distance between two consecutive R-peaks. The variance in time between two consecutive R-peaks reflects the status of the ANS, since HRV is regulated by the sympathovagal balance. RR interval decreases while a person is in a high stress or arousal situation.

Cognitive impairment with arousal: Though memory loss is the primary symptom of dementia, it can highly affect the arousal pattern of the individuals during daily activities. There are three types of arousal [5]. (i) Cognitive arousal is associated with thinking and stimulation while exploring and learning things, such as listening to instructions from the instructor, (ii) Affective arousal is associated with emotional sensation, such as anger, joy, scare, excitement etc., and (iii) Physical arousal is associated with bodily activation while the person is engaged with different daily activities. Persons with dementia have a wide range of arousal responses based on the task they perform in their everyday environment. While persons with Alzheimer's disease (AD) may result in higher arousal than older adults without dementia in performing daily activities, behavioral variant frontotemporal dementia (bvFTD) may reflect emotional blunting with reduced arousal even less than normal older adults [4]. Persons with mild cognitive impairment (MCI) reflect different arousal in different situations. However, insight and awareness about the task difficulty is one of the major reasons for physical arousal. Recent studies have shown that social engagement and the awareness of harder tasks can highly affect mental arousal [36]. People who are aware of harder tasks and successfully complete these tasks with their intellectuals show high arousal (physical arousal) in comparison to people who experience difficulty with the tasks but are not explicitly aware of their challenges. This important finding can be an indicator for dementia symptom analysis for older adults.

Cognitive impairment with activity performance: The effects of cognitive ability on daily activity performance have been studied by prior researchers [14], [16]. They showed that cognitive impairment highly reduced the daily activity performances and this activity performance can be computed as an indicator of cognitive ability status of older adults. The activity performances refer to completeness of task, sequential task ability, interruption avoidance capabilities etc.

Relying on the above insights, we designed ambient and wearable technology assisted computational methodologies and contrasted them with existing clinically-driven survey and observation-based assessment tools to continuously monitor physical and mental changes over functional and behavioral activities of older adults to help them aging-in-place.

\section{RELATED WORKS}

A smart home is a evolving computing environment where multimodal sensor technologies are deployed and overlayed with a variety of computational techniques to reason about and control the physical home setting. The sensor events are normally being generated while participants perform their daily activities. Prior research has demonstrated that smart home is an ideal environment for providing automated health monitoring and assessment to the aging population. Pavel et al. [16] showed that change in mobility patterns are related to change in cognitive ability. Lee [15] designed an embedded sensing system to increase awareness of functional abilities in the older population. These techniques help map a sequence of readings from a particular sensor modality to a label indicating the activity that is being performed. In our deployment, we built a minimally non-invasive easy-to-install smart home system consisting of ambient infrared motion detectors and wearable wristworn sensors (accelerometer, electrodermal activity, photoplethysmography and skin temperature) to gather information about inhabitants' complex activities, such as sandwich making, sweeping, tooth brushing, eating etc., in conjunction with physiological signals such as stress, anxiety, depression and heart rate [21].

Several approaches have been proposed recently to recognize mental arousal level; some of them are based on physiological signals such as blood pressure [22], heart rate [22], heart rate variability (HRV) [24], skin conductance, cortisol [27], pupil diameter [31]. Activity of sympathetic and parasympathetic nervous system can be monitored through blood pressure, heart rate and HRV [22]. Hernandez [26] articulated the inverse relationship between self-ratings of perceived emotional stress and high frequency component $(0.15-0.5 \mathrm{~Hz})$ of HRV. Skin conductance has been considered as another biomarker for stress detection, where eccrine sweat activity that is controlled by only sympathetic nervous activity is measured. [26] discriminated stressful and non-stressful calls at the call center environment using skin conductance features [26]. Setz et al. automatically classified skin conductance responses from cognitive load and stress with accuracy higher than $80 \%$ [32]. They also attempted to classify the same two conditions using seating pressure data and obtained over $70 \%$ accuracy [33]. Autosense proposed wearable sensor suite for inferring onset causality and consequences of stress in the field study [29].

Nowadays there are many wearable devices being instrumented including smart wristwatch, smart phones, smart shoes and biometric sensors to measure physiological and behavioral signals from the users living in their own environment. Even accelerometer sensors have been used for continuous arousal monitoring though they are commonly used for recognizing ambulatory movements (e.g., walking, running, sitting, climbing, and falling). In this paper, we combine daily activities task quality with physiological markers to predict fine-grained stress/anxiety features for different groups of older individuals. Though activity recognition based task quality measurement and correlating with cognitive impairment assessment has been a evolving research problem [3], [14], no prior works explored the cognitive and functional health assessment by combining the task quality and physiological markers. Our proposed framework is inspired by Kumar et al. [28] who 


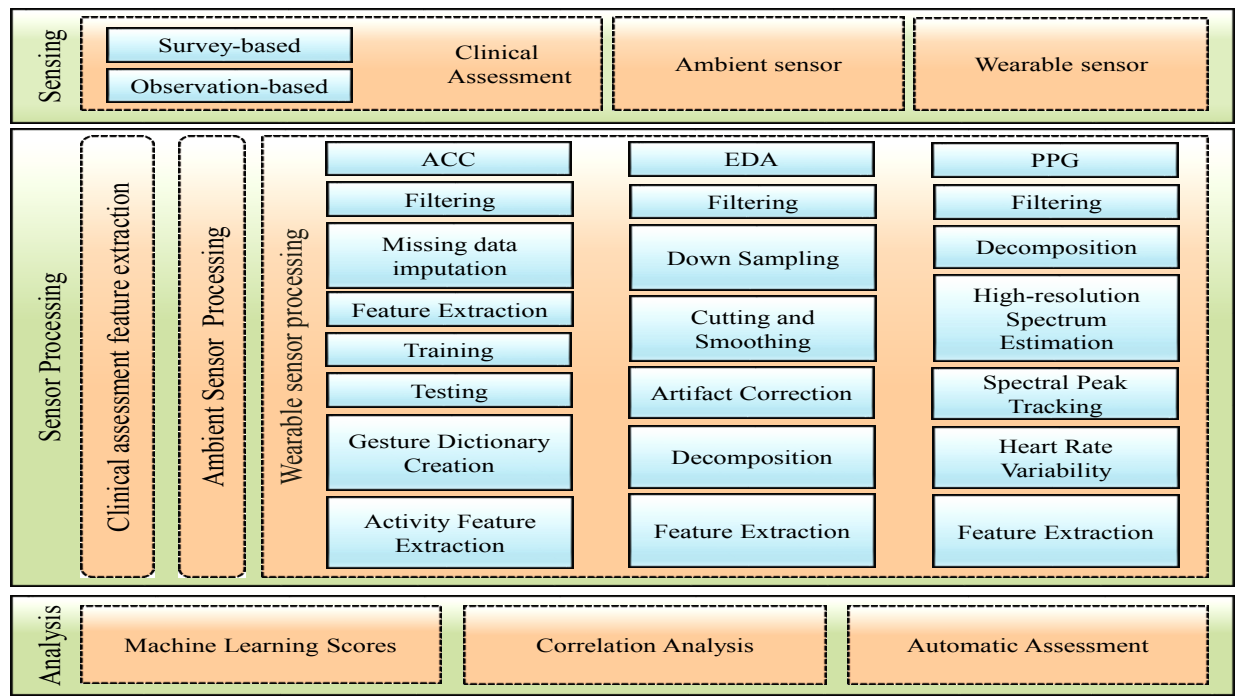

Fig. 1. An Overview of Functional and Behavioral Health Assessment Framework

presented a continuous stress assessment gold standard in mobile environment. We hypothesize that cognitive ability of older adults (normal, mild cognitive impairment, Alzheimer's disease) may affect the physiological markers and arousal activation amplitude along with their daily activity performances. We postulate an unsupervised machine learning approach in scoring daily activity performances and signal processing algorithms on EDA and PPG signal to extract arousal features. We quantify the correlations between clinically observed measures and automated computed features through statistical analysis and machine learning predictions.

\section{Overall Architecture And Study Procedure}

Fig. 1 illustrates the overall architectural view of our proposed framework. The entire framework is comprised of three component modules: (i) sensing, (ii) sensor processing, and (iii) analysis. The sensing module consists of clinical assessment and sensing signals from ambient and wearable sensors. Sensor processing module is comprised of three major sub-modules: a) clinical assessment feature extraction; b) ambient sensor feature extraction; and c) wearable sensor feature extraction (ACC, EDA and PPG). Analysis module is comprised of machine learning score prediction of daily activity performances, quantification of statistical correlation, and automatic assessment analysis sub-modules. Next we discuss the smart home system setup followed by the inclusion criteria of participants and details of our study procedure that we designed to collect data from older adults in a retirement community center.

\section{A. Smart Home Setup}

We developed a real testbed smart home system consisting of customized Cloud Engine PogoPlug Mobile [35] base server, 10 wireless sensor tag (WST) sensors [20] (7 object sensors and 3 PIR sensors), one Ethernet tag manager, one router and one Empatica E4 wrist band (EDA, ACC and PPG sensors). PogoPlug base server is placed in a corner of living room with a continuous power supply which is associated with Ethernet tag manager and a router. 3 binary PIR sensors are placed in three different rooms (kitchen, livingroom and bedroom) and 7 binary object sensors (closet door, entry door, telephone, broom, laundry basket, trash can and trash box). We set the sensor sensitivity at 55\% (best choice tested on trial and error basis) thus slightest vibration on the associated sensor of the object fires without any false alarm. We use three IP cameras in the appropriate positions to collect the ground truth data. Empatica E4 is a multi-sensor wristband capable of measuring different vitals with different fixed sampling frequency. The Photoplethysmography (PPG) sensor measures the Heart Rate and Inter-Beat-Intervals at a $32 \mathrm{~Hz}$ frequency. Skin conductance is measured using the Electrodermal activity (EDA) sensor at $4 \mathrm{~Hz}$ frequency. Body temperature is given by an optical thermometer and a triaxial accelerometer gives the 3 dimensional accelerometer data at a $32 \mathrm{~Hz}$ sampling rate. The sensor values are saved in its internal memory. Ethernet Tag Manager is a low cost Ethernet hub that helps create a bridge between cloud and all wireless sensor tags. Fig. 2 shows our Pogoplug smart home system prototype.

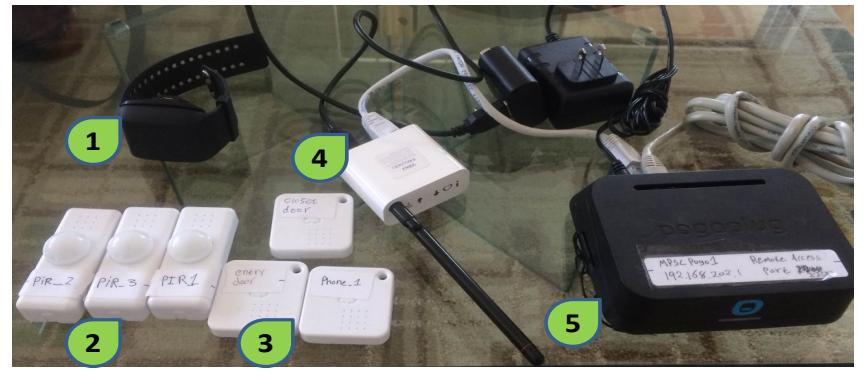

Fig. 2. Pogoplug smart home system devices: (1) Empatica E4 Wristband, (2) Wireless Sensor Tag Passive Infrared Sensor, (3) Wireless Sensor Tag Object Sensor, (4) Ethernet Tag Manager, (5) Cloud Engine Pogoplug Mobile

\section{B. Inclusion Criteria}

Recruitment of participants and data collection occur within one continuing care retirement community in Baltimore City which is supportive of this project and includes independent living and assisted living residents. Older adults are eligible to participate in this study if they are 65 years of age or older $(100 \%$ of the retirement community meet this 
TABLE I. FUnCtional ACtivities

(Index) Description
(1) telephone calling and (2) receiving, (3) sweeping, (4) preparing sandwich,
(5) taking out the trash, (6) folding clothes, (7) combing hair, (8) washing hands,
(9) brushing teeth, (10) putting on a jacket/sweater and (11) shoes, (12) answering
the door, and (13) writing a check.

requirement), speak English, and live in independent living or assisted living at the continuing care retirement community at the time of recruitment. Residents are excluded from the study if they live with another person to avoid multi-inhabitant smart home activity recognition. There are no group assignment and all study participants are exposed to the same study procedures. A research evaluator administers the Evaluation to Sign Consent (ESC), which helps determine the resident's ability to consent participation in the study. Residents who are willing to participate in the study, but are unable to pass the ESC are asked to sign an assent form and evaluator then proceeds to the Legally Authorized Representative to potentially obtain consent on behalf of the resident. Table I shows 13 functional activities that we used in this study.

\section{Demographics, Observation \& Survey Data Collection}

Following resident consent, the clinical research evaluator collects the following data: demographic and descriptive data (age, gender, race, ethnicity, marital status, date of admission to continuing care retirement community, independent living or assisted living setting, education, medical commodities, and prescribed medication). The clinical evaluator has performed two types of clinical assessment; observation or performance based and survey based assessment. For observation based assessment, the resident's cognition is assessed using the Saint Louis University Mental Status (SLUMS) scale [23]. For survey based assessment the following self-report surveys are completed. Functional health assessment are done using Yale Physical Activity Survey [19], Lawton Instrumental Activities of Daily Living, and Barthel Index of Activities of Daily Living [34]. Cognitive health assessment are done based on Geriatric Depression Rating scale [17] and Zung Self-Rating Anxiety scale [18]. All of these survey instruments are widely used and well validated in geriatric research to assess psychometric properties of cognitive and functional health of older adults.

\section{Quantification of Performance Score}

In this section, we discuss the activity and physiological sensor data feature extraction and mapping steps to enumerate the performance measure score that help create the ground for comparing later the technology guided assessment with the clinical evaluation. To characterize both the qualitative and quantitative performance score, we start with four different feature groups ranging from both functional and behavioral health measures as listed below. (a) Observation based activity features, (b) Technology guided automated activity features, (c) Electrodermal activity features, and (d) Heart rate variability features.

\section{A. Observation Based Activity Features}

We design a complex activity set comprises of multiple subtasks which are involved with task concurrency,
TABLE II. MICRO STEPS OF A COMPLEX ACTIVITY NAMELY Preparing Sandwich. EACH MICRO STEP IS INVOLVED WITH HAND GESTURE AS WELL AS OBJECT AND/OR AMBIENT SENSOR FIRINGS

\begin{tabular}{|c|l|c|}
\hline Seq. & Activity & $\begin{array}{c}\text { Plausible } \\
\text { Seq. Switch }\end{array}$ \\
\hline a & Use napkins to clean hands & \\
\hline b & Retrieves a plate, cup, utensil & c \\
\hline c & Retrieves bread from kitchen cupboard & b \\
\hline d & Cuts sandwich in half diagonally & e \\
\hline e & Taking out jam/jelly/butter & d \\
\hline f & Use jam/jelly/butter & e \\
\hline g & Pours drink & $\mathrm{h}$ \\
\hline h & Eat sandwich & \\
\hline i & Drink water & \\
\hline j & Puts napkins in trash & \\
\hline
\end{tabular}

interleaving and interruption. Participants are instructed to perform the scripted activities as listed in Table I while the evaluator observed the aforementioned functional activity performance measures (interleave, interruption, completion, repetition). Each incorrect attempt of performance measure costs one point. For example, 'preparing sandwich' activity consists of 10 subtasks as shown in Table II. Some of the sequences can be swapped without having any negative cost on the performance scores. For example, a sequence of subtasks $\{a, b, c, d, e, f, \ldots, j\}$ scores same as $\{a, c, b, d, e, f, \ldots, j\}$ as ' $b$ ' and 'c' can be swapped without incurring any cost to performance scores. If we consider, one of the subtasks remains incomplete (1 point), two subtasks face interruptions ( 2 points), one task is interleaved by one irrelevant task (1 point) and two tasks are performed twice each ( 2 points), then the final activity performance score is $6(1+2+1+2)$. A higher score reflects lower performance of functional activities. The evaluator also observed the duration of each subtask, capability of doing that particular task and denial of completing. However, taking rest in-between the two tasks does not cost any performance score.

\section{B. Technology Guided Automated Activity Features}

We consider ambient and wearable accelerometer sensors readings combined with machine learning approach for measuring automatic activity performance score. Prior researches [3], [14] proposed a sequence of ambient motion sensor streams as complex activity components. We consider an additional wearable wrist band with in-built accelerometer sensor to detect hand gesture augmenting with the ambient sensor readings to help recognize complex activities.

(1) Accelerometer Feature Extraction: To extract accelerometer feature, we apply a high-pass filter with a cut-off frequency of $4 \mathrm{~Hz}$, splitting each time series data stream into a set of nonoverlapping windows of feature vector through a trial and error method. We use 57 statistical features that have uniaxial (mean, percentiles, signal power, log energy, standard deviation, peak intensity etc.) and biaxial (correlation coefficient feature, lag one autocorrelation etc.) statistical properties. We apply correlation feature selection algorithm [42] on the computed 57 features to select 12 best features for classification of hand gesture.

(2) Hand Gesture Classification: We hypothesize that every functional activity consists of a sequence of hand gestures unlike just the locomotive motion classification [3], [14]. To build 


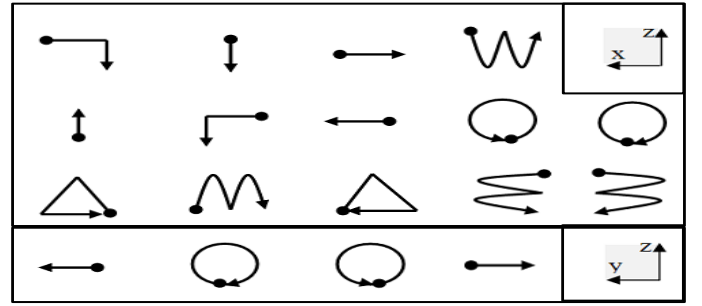

Fig. 3. Hand gesture dictionary of 18 gestures with their axis of direction

a gesture dictionary, we collect 18 hand gestures (standard hand gesture dictionary stated in [37]) from 5 participants over an hour of session each. We extract the accelerometer features, train a Support Vector Machine based SMO algorithm [25], and test with a 10 -fold cross validation. We apply the gesture dictionary based prediction algorithm on the accelerometer sensor extracted features from 17 older adults to recognize the complex activities. Fig. 3 shows 18 hand gesture symbols with their corresponding axis notation.

(3) Supervised Scoring: Initially, we formulate the automated scoring problem as a supervised machine learning problem in which machine learning algorithms learn a function that maps $<$ hand gesture, ambient sensor $>$ feature set to the direct observation scores. We use bagging [12] ensemble method to learn the mapping function and support vector machine (SVM) with sequential machine optimization [25] as base classifier. The bagging improves performance of an ensemble learning algorithm by training the base classifiers on randomly-sampled data from the training set. The learner averages individual numeric predictions to combine the base classifier predictions and generates an output for each data point that corresponds to the highest-probability label.

(4) Unsupervised Scoring: We derive unsupervised scores using a dimensionality reduction (Principal Component Analysis) technique by reducing high dimensional dataset to one with a lower dimension [30]. We use this to reduce the feature set to a single numeric score. Finally, min-max normalization is applied on reduced features that provides us a uniform range of the variables using the following equation:

$$
z_{i}=\frac{x_{i}-\min (x)}{\max (x)-\min (x)}
$$

where $x=\left\{x 1, \ldots, x_{n}\right\}$ and $z_{i}$ is $i^{\text {th }}$ normalized data.

\section{Physiological Signal Processing fOR BEHAVIORAL HEALTH}

We discuss how physiological sensor data can be processed using signal processing techniques to extract the relevant features in presence of users inherent movements and motion artifacts. We carefully choose the specific features which we can define as direct effects of mental arousal.

\section{A. Electrodermal Feature Extraction}

Electrodermal activity also known as skin conductance measurement over time includes two components. (i) Skin conductance Base Level (SBL), which changes slowly over time (tonic changes) and indicates the general activation of
TABLE III. Continuous Decomposition ANALysis FeAtures

\begin{tabular}{|c|c|}
\hline CDA & Description \\
\hline nSCR & Number of significant SCRs within response window (wrw) \\
\hline Latency & Response latency of first significant SCR wrw \\
\hline AmpSum & Sum of SCR-amplitudes of significant SCRs wrw \\
\hline SCR & Average phasic driver wrw \\
\hline ISCR & Area (i.e. time integral) of phasic driver wrw \\
\hline PhasicMax & Maximum value of phasic activity wrw \\
\hline Tonic & Mean tonic activity wrw \\
\hline
\end{tabular}

TABLE IV. Discrete Decomposition Analysis Features

\begin{tabular}{|c|l|}
\hline DDA & Description \\
\hline nSCR & Number of significant SCRs within response window (wrw) \\
\hline Latency & Response latency of first significant SCR wrw \\
\hline AmpSum & Sum of SCR-amplitudes of significant SCRs wrw \\
\hline AreaSum & Sum of SCR-area of significant SCRs wrw \\
\hline Tonic & Mean tonic activity wrw \\
\hline
\end{tabular}

the sympathetic nervous system, (ii) Skin Conductance Responses (SCRs), changes that last for shorter periods (phasic changes). SCRs indicate the activation of the somatic nervous system (SNS) but also reflect responses to events that are new, unexpected, relevant, and/or aversive. Using EDA data to measure arousal in a continuous stimulus setting requires three steps in data processing and analysis. First step is pre-processing which involves data cleaning, filtering, downsampling, cutting, smoothing, artifact correction and decomposition of the signal into its tonic and phasic components. The SBL is typically approximated by frequency filtering, statistical modeling or simple linear interpolation between the skin conductance measures that are not overlaid by responses. The second step is parameterization, which involves deciding which parameter of the EDA data to measure/calculate. For a phasic parameter, this process includes massive abstraction of the phasic signal component, for example, counting responses. The third step is the correlation of the extracted data with the stimulus. We used LedaLab [40] toolbox for EDA data preprocessing and extracting features. We employed butterworth low-pass filter, hanning smoothing with window size 4 and manual movement artifact correction. We decomposed EDA data into its tonic and phasic components using Continuous Decomposition Analysis (CDA) and Discrete Decomposition Analysis (DDA) as discussed below.

(1) Continuous Decomposition Analysis (CDA): This method helps extract the phasic (driver) information underlying the EDA signal, and aims at retrieving the signal characteristics of the underlying sudomotor nerve activity (SNA). EDA data is deconvolved by the general response shape which results in a large increase of temporal precision and then data is being decomposed into continuous phasic and tonic components [38]. This helps compute the several standard features of phasic EDA. We tracked the related events as our pre-labeled activities and extracted 7 time-domain features from CDA as shown in Table III. We used standard deviation, mean and variances on these features over the complex activity classification. Fig. 4(a) shows different decomposition steps of CDA method of a single activity episode.

(2) Discrete Decomposition Analysis (DDA): This method decomposes EDA data into distinct phasic components and a tonic component by means of Nonnegative Deconvolution. The method helps capture and explore all intra-individual de- 


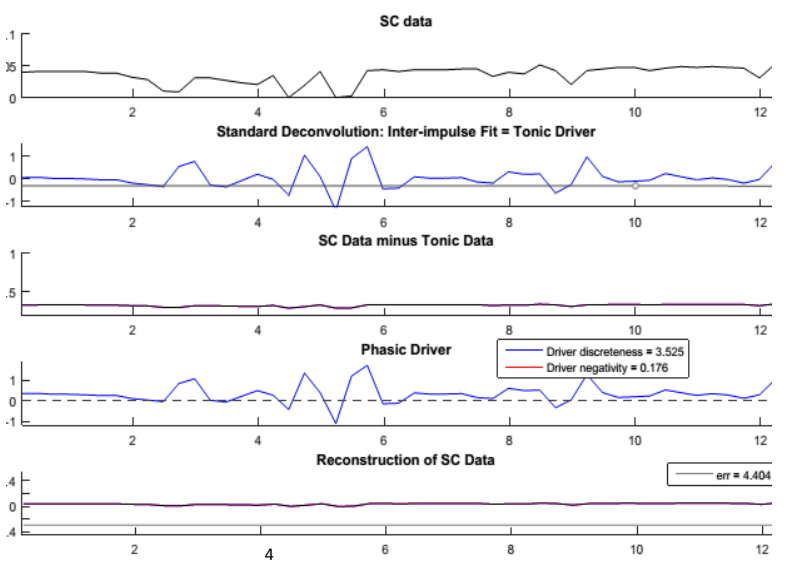

(a)

Fig. 4. (a) CDA decomposition steps (b) DDA decomposition steps

viations of the general response shape and compute a detailed full model of all components in the entire data set [39]. This method is particularly suited for physiological models of the SCR. We extracted 5 features from DDA as shown in Table IV for each activity event and extracted statistical mean, variance and standard deviation on these over the activity session. Fig. 4(b) shows different decomposition steps of DDA method of a single activity episode. The decomposition results in the extraction of distinct response components and thus allows for an unbiased quantification of Skin Conductance Response (SCR) characteristics (e.g., SCR amplitude). We extracted total $12 \times 3=36$ features from decomposition that are subject to be proportional to mental arousal [38], [39].

\section{B. Heart Rate Variability Feature Extraction}

We obtained instant heart rate (HR) from the Empatica E4 wristband provided API which uses a pulse oximeter sensor photoplethysmographic (PPG). PPG is used mainly for measuring the oxygen saturation in the blood and blood volume changes in skin. The Empatica E4 API provides Heart Rate Variability (HRV) i.e., inter-bean interval (IBI) which removes the wrong beats caused by motion artifacts resulting a lot of missing IBI values. However, analysis of real time arousal monitoring needs continuous IBI values for each time stamp. To tackle this we used real time correction method of heart inter-beat intervals [43]. We applied two types of feature extraction method on the corrected heart rate (HR) measure. (i) Time-domain method: This is the simplest method to perform since it can be applied straight to the series of successive RR interval values; (ii) Frequency-domain method: a power spectrum density (PSD) estimate is calculated for the RR interval series. The regular PSD estimators implicitly assume equidistant sampling and thus, the RR interval series is converted to equidistantly sampled series by interpolation methods prior to PSD estimation. In this work, we used a cubic spline interpolation method. The HRV spectrum is calculated with FFT based Welch's periodogram method and with the Autoregression (AR) method [13]. In the Welch's periodogram method the HRV sample is divided into overlapping segments. The spectrum is then obtained by averaging the spectra of these segments. This method decreases the variance of the FFT

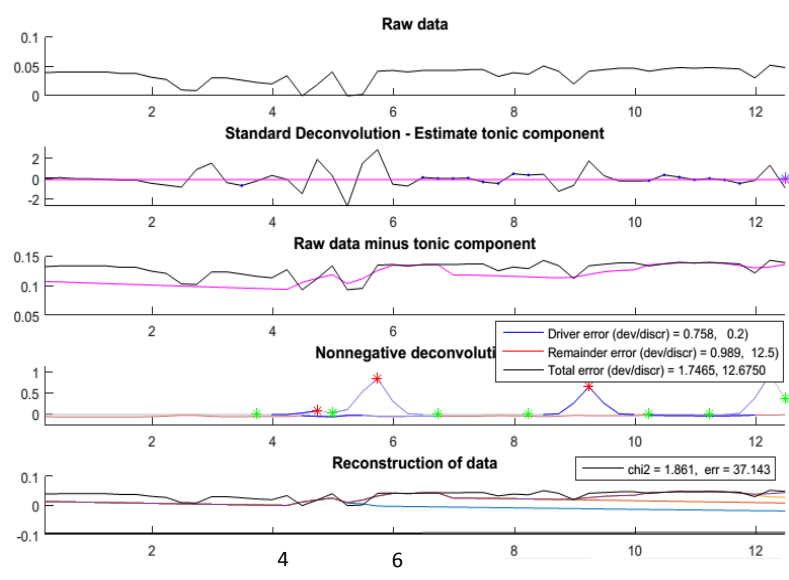

(b)

TABLE V. TIME-DOMAIN HEART Rate VARIABILITy FEATURES

\begin{tabular}{|c|l|}
\hline Feature & Description \\
\hline$\overline{R R}$ & Mean RR intervals \\
\hline SDNN & Standard deviation of RR intervals \\
\hline SDSD & Std of successive RR interval differences \\
\hline RMSSD & Root mean square of successive differences \\
\hline NN50 & \#successive intervals differing more than $50 \mathrm{~ms}$ \\
\hline pNN50 & relative amount of NN50 \\
\hline HRVTI & Total number of RR intervals/height of the histogram \\
\hline TINN & Width of RR histogram through triangular interpolation \\
\hline
\end{tabular}

TABLE VI. FREQUENCY-DOMAIN HEART RATE VARIABILITY FEATURES

\begin{tabular}{|c|l|}
\hline Feature & Description \\
\hline Peak frequency & VLF, LF, and HF band peak frequencies \\
\hline Absolute power & Absolute powers of VLF, LF, and HF bands \\
\hline Relative power & Relative powers of VLF, LF, and HF bands \\
\hline Normalized power & Powers of LF and HF bands in normalized units \\
\hline LF/HF & Ratio between LF and HF band powers \\
\hline
\end{tabular}

spectrum. The generalized frequency bands in case of shortterm HRV recordings are the very low frequency (VLF, 00.04 $\mathrm{Hz}$ ), low frequency (LF, $0.040 .15 \mathrm{~Hz}$ ), and high frequency (HF, $0.150 .4 \mathrm{~Hz}$ ). The frequency-domain measures extracted from the PSD estimate for each frequency band include absolute and relative powers of VLF, LF, and HF bands, LF and HF band powers in normalized units, the LF/HF power ratio, and peak frequencies for each band. In the case of FFT spectrum, absolute power values for each frequency band are obtained by simply integrating the spectrum over the band limits. In the case of AR spectrum, on the other hand, if factorization is enabled distinct spectral components emerge for each frequency band with a proper selection of the model order and the absolute power values are obtained directly as the powers of these components. If factorization is disabled the AR spectrum powers are calculated as for the FFT spectrum. Table V and Table VI show calculated time-domain and frequency-domain features of HRV spectrum which are subject to be inversely proportional to mental arousal. 


\section{EXPERIMENTAL PROCEDURE}

We have recruited 17 participants for this study consists of 15 females and 2 males (similar to overall gender distribution of the retirement community: $85 \%$ female and $15 \%$ male) with age range from 77-93 (mean 85.5, std 3.92) from the participating continuing care retirement community in Baltimore City through the appropriate institutional IRB approval. The experimental setup are discussed below.

\section{A. Methodology}

A study team member at the participating continuing care retirement community helped in recruiting participants who reside in the facility and meet the study eligibility criteria. Informed consent or assent is obtained from the participants in accordance with IRB procedures and a study visit is scheduled for data collection. During the study visit, a trained gerontology graduate student evaluator helps participants to fill out the validated surveys. Participants are given an Empatica E4 to wear on their dominant hand, and concurrently another trained Information Systems graduate student have the PogoPlug smart home setup in their own living environment. The participants are instructed to perform the scripted activities with the presence of only the evaluator. Another project member remotely monitors the sensor readings, videos and system failure status. Evaluator computes the activity performance score (i.e., completion of the task, duration, and sequencing) and all the surveys as discussed previously. The entire session varies from 1 to 2 hours of time depending on an individual participant's physical and cognitive ability to perform activities.

\section{B. Ground Truth Annotation}

The recorded videos are used to annotate ground truth of activities. Two graduate students are engaged to annotate the activities, and the ambient sensor firings whereas the activity performances are computed by the evaluator. Two more graduate students are engaged to validate the annotations. The recorded videos are stored in a secured UMBC server and immediately destroyed in accordance with the IRB protocol.

\section{EVALUATION}

We evaluate our proposed multimodal functional and behavioral health assessment framework consisting of wearable and ambient sensors to help achieve the automated assessment of task quality, cognitive measures and their underpinning inter-correlations and statistical significance with well established clinical-driven survey and observation based assessment tools and techniques. We divide our participants pool into several groups based on the clinical assessment score as shown in Table VII. The feature subsets used in the experimentation for observation and survey based clinical assessments and technology guided physiological (behavioral) and activity (functional) initiated health assessments are depicted in Table IX. To analyze correlations, we used Pearson correlation coefficients with significance on $p<0.05$.

\footnotetext{
${ }^{*} p<0.05$ with Bonferroni correction for 17 samples

** $p<0.005$ with Bonferroni correction for 17 samples

$\dagger$ Not Significant
}

TABLE VII.

PARTICIPANT GROUPS

\begin{tabular}{|c|c|}
\hline Description & Count \\
\hline $\begin{array}{c}\text { No Cognitive } \\
\text { Impairment (NCI) }\end{array}$ & 8 \\
\hline $\begin{array}{c}\text { Mild-Cognitive } \\
\text { Impairment (MCI) }\end{array}$ & 3 \\
\hline $\begin{array}{c}\text { Cognitively } \\
\text { Impaired (CI) }\end{array}$ & 6 \\
\hline
\end{tabular}

TABLE VIII. GROUP CORRELATIONS

\begin{tabular}{|c|l|l|l|}
\hline Groups & INT & EDA & HRV \\
\hline $\begin{array}{c}\text { NCI, MCI } \\
\text { CI }\end{array}$ & $0.638^{*}$ & $-0.334^{\dagger}$ & $0.494^{\dagger}$ \\
\hline NCI, MCI & $0.449^{\dagger}$ & $0.51^{*}$ & $-0.51^{*}$ \\
\hline MCI, CI & $0.469^{\dagger}$ & $-0.53^{*}$ & $0.464^{\dagger}$ \\
\hline NCI, CI & $0.484^{\dagger}$ & $-0.58^{*}$ & $0.69^{*}$ \\
\hline
\end{tabular}

TABLE IX. Feature Subsets

\begin{tabular}{|c|l|}
\hline Feature & Description \\
\hline Observation & $\begin{array}{l}\text { Activity Ability (AA), Sequencing (SEQ), Interruptions (INT), } \\
\text { Duration (DU) }\end{array}$ \\
\hline Survey & $\begin{array}{l}\text { SLUMS Score (S-Score), ZUNG Score (Z-Score), IADL Score } \\
\text { (I-Score), Yale Score (YPAS), Barthel Score (B-Score), } \\
\text { GDS Score (G-Score) }\end{array}$ \\
\hline Physio: EDA & CDA (7 features), DDA (5 features) \\
\hline Physio: HRV & Time Domain (TD) 8 features, Frequency Domain (FD) 5 features \\
\hline $\begin{array}{c}\text { Activity: Machine } \\
\text { Learning }\end{array}$ & Supervised (AA, SEQ, INT), Unsupervised (AA, SEQ, INT) \\
\hline
\end{tabular}

TABLE X. INDIVIDUAL FEATURE CORRELATIONS

\begin{tabular}{|l|l|l|l|l|}
\hline & YPAS & AA & B-score & I-score \\
\hline SEQ & $0.553^{*}$ & $0.962^{* *}$ & $0.202^{\dagger}$ & $0.475^{\dagger}$ \\
\hline EDA & $0.225^{\dagger}$ & $0.349^{\dagger}$ & $-0.519^{*}$ & $-0.53^{*}$ \\
\hline
\end{tabular}

\section{A. Observation-based Feature Correlations}

No significant correlations are noted in case of physiological health (i.e., anxiety and depression) assessment in the three groups (NCI, MCI, and CI) of dementia patients, but the cognitively impaired (CI) group shows a significant correlation with INT score (coefficient $0.638 *$ ) (Table VIII). This depicts that as the dementia status gets worse, the participants' functional activities are interrupted more frequently in comparison to NCI participants. Older adults experiencing frequent interruptions while performing everyday activities delineate the fact that they are less aware of the task properties. On the contrary, it can be postulated that the NCI individuals are remained aware of the task they perform. The awareness degrades as their dementia status goes worse, MCI to CI, which posits significant degradation in the task interruption performance. However, significant correlations are noted respectively between SEQ score and YPAS $\left(0.553^{*}\right)$, SEQ score and AA $(0.962 * *)$ (Table X). These correlations demonstrate that the task sequencing ability depends on the participants' functional abilities to perform a specific task. Fig. 5(d) presents a scatter plot of bivariate correlations between different dementia groups (NCI, MCI and $\mathrm{CI}$ ) with observation-based interruption scores along the fitting line.

\section{B. Electrodermal Feature Correlations}

We extracted $7 \mathrm{CDA}$ and 5 DDA features from EDA signals. EDA signal helps measure the intensity and amplitude of arousal while the daily activities are being performed. If the group correlations between the independent EDA features variables and dependent Dementia group variables (NCI, MCI, CI) positively correlates, that means, individual group's arousal state increases as dementia intensity increases. If it negatively correlates, then arousal decreases as dementia intensity increases. For dementia group segmentation, we first consider 


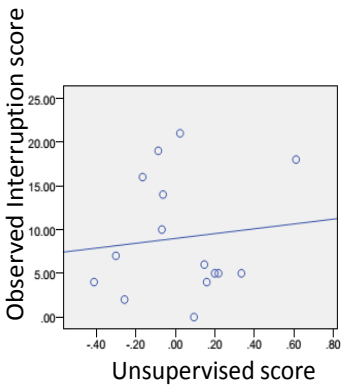

(a)

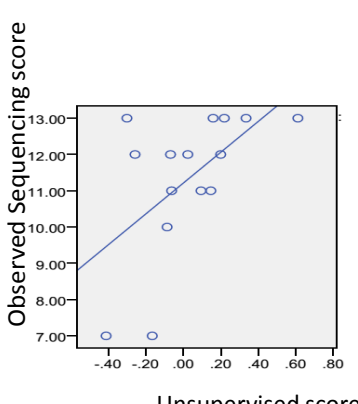

(b)

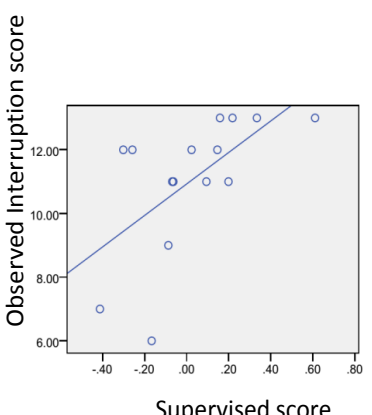

(c)

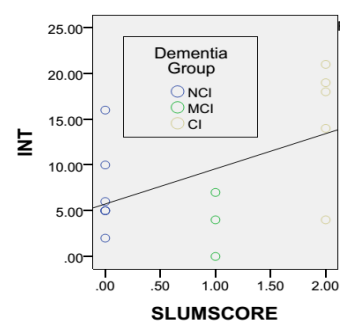

(d)

Fig. 5. Scatter plot of bivariate correlations between calculated activity performance score vs. observed scores with fitting line. (a) unsupervised vs. observed interruption scores; (b) unsupervised vs. observed sequencing score; (c) supervised vs. observed interruption score; (d) observed interruption vs. dementia group correlations

$\mathrm{NCI}$ and MCI, and note that they are highly correlated with EDA features $\left(0.51^{*}\right)$ which means MCI individuals aroused more than NCI individuals while performing activities. Considering NCI and CI, we observe that they are negatively correlated $\left(-0.58^{*}\right)$ which means $\mathrm{CI}$ individuals show less arousal than NCI. While considering MCI and CI, we note that they are negatively correlated with EDA features $\left(-0.53^{*}\right)$ which means $\mathrm{MCI}$ groups show more arousal than $\mathrm{CI}$ individuals. We also note that B-Score and I-Score are negatively correlated with EDA features $\left(-0.519^{*}\right.$ and $\left.-0.53^{*}\right)$ which means the most functional active individuals show more arousal while performing activities (Table VIII).

\section{Heart Rate Feature Correlations}

Mental arousal (stress, anxiety etc.) is possible reason to decrease Heart Rate Variability features (increase of heart rate). We extracted 8 time domain and 5 frequency domain heart rate variability (HRV) features over the activity performance period. Considering NCI and MCI groups, we note a significant negative correlations $\left(-0.51^{*}\right)$ reflecting that $\mathrm{MCI}$ group may have more arousal than NCI while performing functional activities. Similarly, considering NCI and CI groups of individuals, we note a significant positive correlations $\left(0.69^{* *}\right)$ delineating that CI group may have less arousal than NCI group (Table VIII). Fig. 5(a), Fig. 5(b) and Fig. 5(c) represent the scatter plots of unsupervised and supervised activity performances scores (both sequencing and interruption score) correlations with the observation-based scores along the fitting line.

\section{Machine Learning Based Activity Performance Correlation}

Automatic activity task quality performance estimation has been investigated previously [14] which achieved sequencing and interruption scores with $0.47^{*}$ and $0.50^{*}$ correlation coefficients respectively for supervised task quality scoring. They also achieved $0.57^{*}$ correlation coefficient for unsupervised task quality estimation. Prior research achieved significant performance correlations with supervised and unsupervised task quality estimation with observed scores considering a sequence of motion sensor streams as components of each complex activity. We additionally consider hand gesture streams along with the motion and object sensor which helps improve the prior correlations significantly.

For machine learning based classification, we first build hand gesture dictionary with 18 gestures (Fig. 3) collecting accelerometer sensor readings from 5 individuals. We then extract accelerometer features, train and test using Random
Forest algorithm and finally 10 -fold cross validation provides us $83.5 \%$ hand gesture recognition accuracy (FP rate $5.1 \%$, Precision $91.5 \%$ and recall $83.5 \%$ ). We then use the hand gesture dictionary and ambient sensor readings, train Random Forest algorithm, which provides us $91.8 \%$ (FP rate 3.5\%, Precision $95.7 \%$ and recall $91.8 \%$ ) accuracy of high-level activity recognition. We apply supervised activity scoring (SVM classifier with SMO algorithm) method and find significant correlations with observed activity performances $\left(0.71^{* *}\right.$ and $0.81^{* *}$ for SEQ and INT scores respectively). We use dimensionality reduction (PCA) on $<$ hand gesture, ambient sensor $>$ feature maps to generate two dimensional feature values. We find significant correlations with the unsupervised feature sets with SEQ and INT scores $\left(0.568^{*}\right.$ and $0.519^{*}$ respectively). We observe that our approach significantly improves the prior proposed method [14].

\section{Discussion}

Mental arousal is a convoluted physiological response of stress and task awareness observed during the performance of everyday activities triggering the increase in EDA and decreases in heart rate signals [36]. The older individuals with increasing arousal suggests that their mental health may have been dominated by stress as well as increasing awareness of the task being performed. On the other hand, decreasing arousal means they are less aware of the task difficulty, even when they have been making mistakes. Our evaluations in the retirement community suggest that persons with MCI show higher arousal (increasing EDA and decreasing heart rate) than cognitively intact (NCI) and cognitively impaired (CI) older individuals. Mild cognitively impaired individuals go through a transition period between normal and dementia. Though the activity performances degrades due to their memory loss, their awareness of the mistakes increases the arousal amplitude (based on the heart rate and EDA signals) even more than the $\mathrm{NCI}$ individuals. The possible reasons for their extreme stress is that, $\mathrm{MCI}$ individuals face more difficulty in completing the task, even if they are not committing any mistakes. Since they are in a transition period, so they may have some symptoms of dementia, such as forgetting the task sequences and thus triggering many interruptions. As MCI individuals are not fully cognitively impaired, they are still aware of their mistakes which apparently increases their arousal state. Persons with CI have more difficulty in performing activities in comparison to persons with MCI and NCI though they may experience a lower rise in mental arousal features. This may be explained in 
part because persons with dementia (CI) are not aware of their mistakes at all due to their cognitive health decline. Therefore, less awareness of their mistakes makes them less aroused in performing tasks of all types as attested in our correlation analysis results.

\section{Conclusion}

Cognitive impairment status can significantly impact mental arousal on everyday activities and as well as activity performances. However, mental arousal can be considered as an important feature of context awareness of daily activities. We propose a hybrid approach combining both physiological and functional health measures by incorporating heart rate, electrodermal activity and daily activity performance monitoring to investigate how cognitive status impacts on these modalities and helps advance the technology assisted automated health assessment. Our hand gesture combined with ambient sensor streams based methodology helps improves automatic complex activity performances estimation significantly. Our study in a real continuing care retirement community demonstrates objective signs of stress with increased heart rate and galvanic skin response in the individuals with MCI. The individuals with dementia have trouble with some of the activities, but have less insight and awareness into their deficits and so are less stressed. The individuals without cognitive impairment are able to do activities easily and then they have only the normal stress response called physical arousal response. We believe the findings in this work are promising and with our ongoing effort on recruiting more participants may help strengthen all the statistical analysis and conjectures. In future, we will apply context aware recommender system that can be used to send alert requesting to remove wrist band in bathroom activities, recommending to wear it in normal activities, sending alarm to caregivers in critical situation, fall/near fall detection and emergency call opportunity only using gesture etc.

\section{ACKNOWLEDGMENTS}

This work is supported by UMB-UMBC Research and Innovation Partnership grant.

\section{REFERENCES}

[1] Alzheimer's disease facts and figures, Alzheimer's \& Dementia, 2012.

[2] A. Abbott Dementia: A problem for our age. Nature 475, 2011.

[3] A. Akl, B. Taati, A. Mihailidis, Autonomous Unobtrusive Detection of Mild Cognitive Impairment in Older Adults. IEEE Trans. Biomed. Engineering, 2015

[4] A. Joshi, J. P. Barsuglia, M. J. Mather, E. E. Jimenez, J. Shapira, M. F. Mendez, Evaluation of emotional blunting in behavioral variant frontotemporal dementia compared to Alzheimer's disease, PMC, 2014.

[5] W. Cannon. Bodily Change in Pain, Hunger, Fear and Rage: An Account of Recent Research into the Function of Emotional Excitement. Appleton, NY, USA, 1915.

[6] U. Lundberg and et al. Psychophysiological stress and EMG activity of the trapezius muscle. International Journal of Behavioral Medicine, 1994.

[7] R. Fortinsky and J. Wasson, How do physicians diagnose Dementia? Evidence from clinical vignette responses, Am. J. Alzheimer's Disease, vol. 12, no. 2, 1997

[8] F. Sun, C. Kuo, H. Cheng, S. Buthpitiya, P. Collins, M. L. Griss, Activity-Aware Mental Stress Detection Using Physiological Sensors, MobiCASE 2010.

[9] F. Martini, E. Bartholomew, Essentials of Anatomy \& Physiology. San Francisco: Benjamin Cummings, 2001

[10] N. Carlson, Physiology of Behavior. New Jersey: Pearson Education, Inc. 2013.

[11] J. Choi, R. Gutierrez-Osuna, Using Heart Rate Monitors to Detect Mental Stress, BSN 2009.

[12] Leo Breiman, Bagging Predictors, Machine Learning 24(2), 1996

[13] B. P. Donald, A. T. Walden, Spectral Analysis for Physical Applications. Cambridge University Press, 1993.
[14] P. Dawadi, D. Cook, and M. Schmitter-Edgecombe. Automated cognitive health assessment using smart home monitoring of complex tasks. IEEE Transactions on Systems, Man, and Cybernetics: Systems, 2013.

[15] M.L. Lee and A.K. Dey, Embedded assessment of aging adults: A concept validation with stake holders, PervasiveHealth, 2010.

[16] M. Pavel, A. Adami,M. Morris, J. Lundell, T.L. Hayes, H. Jimison, and J.A. Kaye, Mobility assessment using eventrelated responses, in Proc. Transdisciplinary Conference on Distributed Diagnosis and Home Healthcare, 2006.

[17] J.A. Yesavage, T.L. Brink, Rose TL, et al. Development and validation of a geriatric depression screening scale: a preliminary report. J Psychiatr Res, PMID 1982-83

[18] Zung WWK. A rating instrument for anxiety disorders, Psychosomatics, 1971.

[19] R.D. Starling, D.E. Matthews, P.A. Ades, E.T. J. Poehlman, Assessment of physical activity in older individuals: a doubly labeled water study, Appl. Physiol, 1999.

[20] Wireless Sensor Tags http://wirelesstag.net/

[21] J. Alon, V. Athitsos, Q. Yuan, and S. Sclaroff, A unified framework for gesture recognition and spatiotemporal gesture segmentation, IEEE Transactions on Pattern Analysis and Machine Intelligence, 2008.

[22] T. G. Vrijkotte, L. J. van Doornen, and E. J. de Geus, Effects of work stress on ambulatory blood pressure, heart rate, and heart rate variability., Hypertension, vol. 35, no. 4, Apr. 2000.

[23] D. F. Tang-Wai, D. S. Knopman, Y. E. Geda, S. D. Edland, G. E. Smith, R. J. Invik, Comparison of the Short Test of Mental Status and the Mini-Mental State Examination in mild cognitive impairment. Archives of Neurology 60 (12), 2003.

[24] R. K. Dishman, Y. Nakamura, M. E. Garcia, R. W. Thompson, A. L. Dunn, and S N. Blair, Heart rate variability, trait anxiety, and perceived stress among physically fit men and women, Int'l Journal of Psychophysiology, vol. 37, no. 2, Aug. 2000.

[25] S. L. Cao, S. Keerthi, C. J. Ong, P. Uvaraj, X. J. Fu, H. P. Lee, Developing parallel sequential minimal optimization for fast training support vector machine, Neurocomputing, 2006.

[26] J. Hernandez; R. R. Morris; R. W. Picard, Call center stress recognition with person-specific models, ACII, 2011

[27] S. S. Dickerson and M. E. Kemeny, Acute stressors and cortisol responses: a theoretical integration and synthesis of laboratory research., Psychological bulletin, vol. 130, no. 3, May 2004.

[28] K. Hovsepian, M. al'Absi, E. Ertin, T. Kamarck, M. Nakajima, S. Kumar, cStress: towards a gold standard for continuous stress assessment in the mobile environment, UbiComp 2015

[29] E. Ertin, N. Stohs, S. Kumar, A. Raij, M. al'Absi, S. Shah, AutoSense: unobtrusively wearable sensor suite for inferring the onset, causality, and consequences of stress in the field, SenSys 2011.

[30] L. Maaten, E. Postma, and H. Herik, Dimensionality reduction: A comparative review, Tilburg University Technical Report, TiCC-TR, vol. 10, 2009.

[31] F. Mokhayeri, M.-R. Akbarzadeh-T, and S. Toosizadeh, Mental stress detection using physiological signals based on soft computing techniques, ICBME, 2011

[32] C. Setz, B. Arnrich, J. Schumm, R. La Marca, G. Trster, and U. Ehlert, Discriminating stress from cognitive load using a wearable EDA device., IEEE transactions on information technology in biomedicine, vol. 14, no. 2, Mar. 2010.

[33] B. Arnrich, C. Setz, R. La Marca, G. Trster, and U. Ehlert, What does your chair know about your stress level?, IEEE transactions on information technology in biomedicine, a publication of the IEEE Engineering in Medicine and Biology Society, vol. 14, no. 2, Mar. 2010.

[34] K. Krapp. G. Group, Activities of Daily Living Evaluation, Encyclopedia of Nursing \& Allied Health, Enotes Nursing Encyclopedia, 11 Oct, 2007.

[35] PogoPlug: http://mpsc.umbc.edu/projects-2/a-sensor-technology-box-for-smarthealth/

[36] J. Hernandez, I. Riobo, A. Rozga, G. D. Abowd, R. W. Picard, Using electrodermal activity to recognize ease of engagement in children during social interactions, UbiComp, 2014.

[37] A. Akl, C. Feng, S. Valaee, A Novel Accelerometer-Based Gesture Recognition System. IEEE Transactions on Signal Processing, 2011.

[38] M. Benedek \& C. Kaernbach, A continuous measure of phasic electrodermal activity. Journal of Neuroscience Methods, 2010

[39] M. Benedek \& C. Kaernbach, Decomposition of skin conductance data by means of nonnegative deconvolution, Psychophysiology, 2010.

[40] Ledalab: http://www.ledalab.de/

[41] Empatica E4 Wristband: https://www.empatica.com/e4-wristband

[42] Hall, M. A. Correlation-based Feature Selection for Machine Learning. PhD Thesis, April, 999

[43] J. Rand, A. Hoover, S. Fishel, J. D. Moss, J. Pappas, E. Muth, Real-Time Correction of Heart Interbeat Intervals. IEEE Trans. Biomed. Engineering, 2007. 